\title{
Hybrid Functional Networks for Oil Reservoir PVT Characterisation
}

\author{
${ }^{1}$ Munirudeen A. Oloso ${ }^{\text {a }},{ }^{2}$ Mohamed G. Hassan ${ }^{a},{ }^{3}$ Mohamed B. Bader-El-Den ${ }^{\mathrm{b}},{ }^{4} J_{a m e s}$ M. Buick ${ }^{\mathrm{a}}$

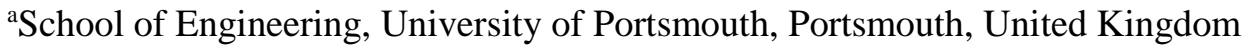 \\ ${ }^{\mathrm{b} S}$ School of Computing, University of Portsmouth, Portsmouth, United Kingdom \\ E-Mails: ${ }^{1}$ munirudeen.oloso@port.ac.uk; ${ }^{2}$ mohamed.hassan@port.ac.uk; ${ }^{3}$ mohamed.bader@ port.ac.uk; \\ 4james.buick@port.ac.uk
}

\begin{abstract}
Predicting pressure-volume-temperature (PVT) properties of black oil is one of the key processes required in a successful oil exploration. As crude oils from different regions have different properties, some researchers have used API gravity, which is used to classify crude oils, to develop different empirical correlations for different classes of black oils. However, this manual grouping may not necessarily result in correlations that appropriately capture the uncertainties in the black oils. This paper proposes intelligent clustering to group black oils before passing the clusters as inputs to the functional networks for prediction. This hybrid process gives better performance than the empirical correlations, standalone functional networks and neural network predictions.
\end{abstract}

Keywords-PVT; API gravity; clustering; functional networks; neural network; black oil

\section{Introduction}

The API gravity $\left(\gamma_{A P I}\right)$ is one of the important pressurevolume-temperature (PVT) properties of crude oils. Crude oil is classified based on this property to determine its heaviness which consequently determines its marketability. Table 1 shows a typical oil classification based on $\gamma_{A P I}$ (Dandekar, 2013; De Ghetto et al., 1995). Knowledge of the $\gamma_{A P I}$ and other PVT properties such as bubblepoint pressure $\left(P_{b}\right)$, oil formation volume factor $\left(B_{o}\right)$ and oil viscosity are important for determining future production or oil reserves from petroleum wells.

Table 1

Crude oil Classification based on API

\begin{tabular}{|l|l|}
\hline Classification & API Range \\
\hline Light & API $>31.1$ \\
\hline Medium & $22.3 \leq$ API $\leq 31.1$ \\
\hline Heavy & API $<22.3$ \\
\hline Extra Heavy & API $<10.0$ \\
\hline
\end{tabular}

Some of the PVT properties, e.g. $\gamma_{A P I}$, can easily be measured or determined onsite while others, e.g. $P_{b}$ and $B_{o}$, are ideally determined through laboratory experimentation. However, this laboratory analysis, which requires special expertise, is expensive and time consuming. Hence, there is a need for a less rigorous, cheaper and quicker solution.

For a long period of time, the petroleum industry has used equations of states (EOS) for determining these PVT properties. However, the EOS are considered computationally complex and require extensive detailed compositions of reservoir fluids. Consequently, many empirical correlations have been developed to meet the industrial demands for less complex, quicker, cheaper and acceptable solutions.

Empirical correlations for predicting PVT properties are generally developed by performing linear or non-linear regression analysis using easily acquired crude oil properties as the inputs. In order to improve the performance of the correlations, some researchers have used $\gamma_{A P I}$ to group crude oils into two or more groups and determine correlations for each group (Kartoatmodjo \& Schmidt, 1994).

For more reliable and improved prediction performance of these PVT properties, other researchers have implemented machine learning [ML] algorithms to predict different PVT properties (Osman \& Al-Marhoun, 2005; Khoukhi et al., 2011; Gharbi et al., 1999; El-Sebakhy et al., 2007). Also, to address the problem in modelling $\mathrm{ANN}$, a recursive least squared algorithm has been used for learning feedfoward ANN to model crude oil blending process (de Jesús Rubio, 2016). However, none of the previous works found in the literature which have applied ML techniques in this field, have taken diversity of API or other input properties into consideration.

For instance, if the data that are used to train an ML algorithm have more light crude oils than heavy oils, then the model is confined to the constraints of the light oils. This type of data is called "imbalanced data set" which is well known in the classification problem (Ramyachitra \& Manikandan, 2014).

This paper proposes a hybrid solution of K-Means clustering and functional networks (FN) for predicting crude oil PVT properties. K-means clustering is used to generate clusters of the input dataset before using functional networks to perform the prediction of the actual target variables, $P_{b}$ and the oil formation volume factor at bubblepoint pressure $\left(B_{o b}\right)$. The performances of the hybrid solution (K-Means+FN) is compared with the standalone FN, artificial neural network (ANN) and selected empirical correlations which are either commonly used in the petroleum industry or recently developed.

The rest of the paper is organised as follows. Section 2 discusses the empirical correlations and ML methods that have 
been used to predict different PVT properties. A brief explanation of K-Means clustering and functional networks in comparison with neural networks is given in section 3 . This is followed by the proposed hybrid solution in section 4 . Results and discussion of the performances of the hybrid model and other compared models are done in section 5 with a brief analysis of sensitivity of FN learning parameters. The conclusion is given in section 6 .

\subsection{Research Contribution}

In this paper, a hybrid of K-Means cluster and $\mathrm{FN}$ has been developed to estimate oil PVT properties. This paves way for intelligent grouping or clustering of crude oils. Also unlike the existing practice where only the $\gamma_{A P I}$ of crude oils is used for grouping them, all the independent variables are used for grouping the crude oils.

\section{Methods for Predicting Oil Reservoir PVT Properties}

Aside the EOS, there are two main categories of methods for predicting oil reservoir PVT properties in the literature: empirical correlations and ML techniques.

\subsection{Empirical Correlations for Predicting Oil PVT Properties}

Many correlations have been developed for the estimation of $P_{b}$ and $B_{o b}$. Usually, the procedure involves linear and/or non-linear regression analyses on the available datasets.

Standing developed graphical correlations for both $P_{b}$ and $B_{o b}$ based on 105 sets of experimental data (Standing, 1947). These graphical correlations were later expressed by equations (Standing, 1977). The input variables that Standing used for the $P_{b}$ correlation are: gas solubility $\left(R_{s}\right)$, gas specific gravity $\left(\gamma_{g}\right)$, $\gamma_{A P I}$ and reservoir temperature (T). For the $B_{o b}$ correlation, the correlating parameters were: $R_{s}, \gamma_{g}$, oil specific gravity $\left(\gamma_{o}\right)$ and $T$. On this basis, many other correlations have been developed for both $P_{b}$ and $B_{o b}$ (De Ghetto et al. 1995; Al-Shammasi 2001; Al-Marhoun, 1988; Vazquez \& Beggs, 1980 ; Almehaideb, 1997; Petrosky Jr \& Farshad, 1998; Jarrahian et al., 2015). It is noted that some of these correlations have replaced $\gamma_{A P I}$ with $\gamma_{o}$ and vice-versa. These two variables are related by equation (1).

$\gamma_{A P I}=\frac{141.5}{\gamma_{o}}-131.5$

\subsection{Machine Learning Techniques for Predicting Oil PVT}

\section{Properties}

The quest for better PVT prediction models to improve the current performances of the empirical correlations has driven applications of different ML techniques in PVT characterisation. The most commonly used ML technique for PVT prediction is artificial neural network (ANN) and its variants (Talebi et al., 2014). While many of the authors have reported good performance from the developed ANN models, others have pointed out that ANN may not necessarily perform better than the empirical correlations (Al-Shammasi, 2001). It should be noted that the performance of any ML model depends on careful selection of its learning parameters.
The "black box" representation of ANN has made it unattractive for adoption in industrial PVT application. Sequel to this, some representative ML and evolutionary techniques have been used, such as support vector machine (SVM), genetic algorithm (GA), adaptive neuro fuzzy system (ANFIS), functional networks (FN) and so on (Khoukhi et al., 2011; ElSebakhy et al., 2007; Hajizadeh, 2007).

Generally, an ML solution usually involves minimization of the error in the learning algorithm. It is notable that many of the machine learning algorithms work by performing a local search that may become stuck in a local minima which causes the model to perform poorly when presented with new data (Dietterich, 2000). An ML model could be stuck in local minima as a result of inefficient learning parameters or imbalanced datasets. However, this problem has not been adequately addressed in prediction of oil PVT properties with ML techniques, though some efforts have been made in empirical correlation development with some possible accuracy improvement (Kartoatmodjo \& Schmidt, 1994). However, this involves manual grouping of crude oils and generating different correlations for the groups. This paper proposes intelligent clustering of data before applying an ML technique to the generated clusters.

\section{Hybrid Machine Learning System}

Hybrid ML system is normally developed so that the consisting sub-systems complement each other. The aim of a hybrid system is to fill the gap that a single method cannot necessarily fill. This paper implements a hybrid of K-means clustering and $\mathrm{FN}$.

\subsection{K-means Clustering}

K-means is a widely used data mining technique. It puts a number of input observations into a number of clusters which must have been defined a priori. There is no defined rule for choosing an optimal number of clusters for a given dataset. The goal of clustering is to allow natural grouping of data (Jain, 2010). The implementation, pros and cons of the K-Means algorithm have been discussed in the literature (Jain, 2010).

\subsection{Implementation of Functional Networks}

FN were introduced as a powerful alternative to neural networks (Castillo, 1998; Castillo et al., 2000). Unlike ANN, FN have the advantage that they use domain knowledge in addition to data knowledge. The network initial topology can be derived based on the modelling of the properties of the real world. Once this topology is available, functional equations allow one to obtain a much simpler equivalent topology.

Several other improvements on the ANN algorithm for different applications can be found in the literature (Chairez, 2016; de Jesús Rubio et al., 2017; Aljarah et al., 2016; Liu et al., 2016).

Simplified general topologies for ANN and FN are shown in Fig. 1 and 2. In these figures, $X_{1}, X_{2}$ and $X_{3}$ are the inputs into the network. $X_{4}$ and $X_{5}$ are the outputs of the hidden layer. $W_{m n}(\mathrm{~m}=4,5,6 ; \mathrm{n}=1,2,3,4,5)$ are the weights while $Y$ is the output in both cases. 


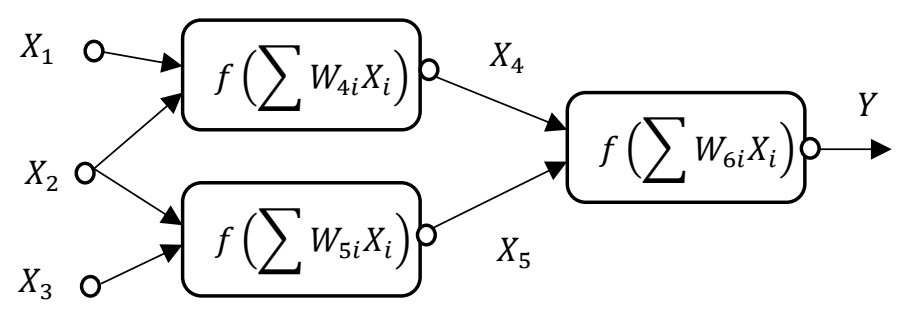

Fig. 1. A Standard Neural Network

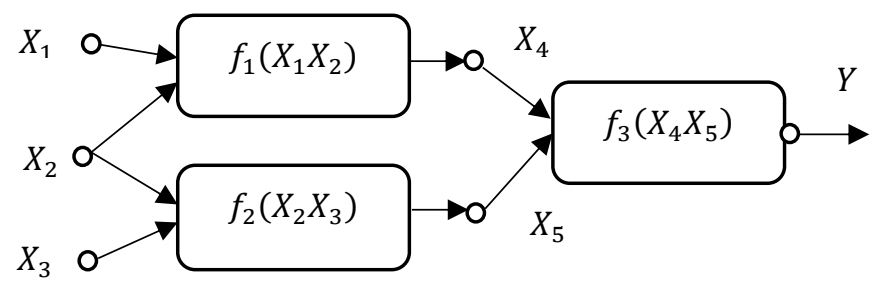

Fig. 2. A Standard Functional Network

There are some quite significant differences between ANN and FN. Notably, the functions in FN are truly learned during the structural learning unlike the ANN where neuron functions are assumed to be fixed and known, and only the weights are learned. The implemented FN is shortly described below.

Given a data set $\left\{x_{i j} \mid y_{i} ; i=1,2, \ldots . n \& j=1,2,3,4\right\}$ where $x_{i j}$ are the predictors and $y_{i}$ is the output. Mathematically, the relationship can be given by

$$
Y=f\left(X_{1}, X_{2}, X_{3}, X_{4}\right)
$$

Note that $j$ refers to the number of inputs which is 4 here. The general form of a functional network that learns from the data can be given as follows (Castillo et al., 2000).

$y_{i}=\sum_{j=1}^{p} \sum_{r=1}^{m} C_{r} \varphi_{r}\left(x_{i j}\right), \quad i=1,2, \ldots, n$

where $\varphi_{r}$ are the linear independent functions which are used to learn the coefficients $C_{r}$. Some possible functions for $\varphi_{r}$ are:

(1). Polynomial function:

$$
\varphi=\left\{1, x, x^{2}, \ldots, x^{m}\right\}
$$

(2). Exponential Function:

$$
\varphi=\left\{1, e^{x}, e^{-x}, \ldots, e^{m x}, e^{-m x}\right\}
$$

(3). Fourier Function:

$$
\varphi=\{1, \sin (x), \cos (x) \ldots, \sin (m x), \cos (m x)\}
$$

4). Logarithm Function:

$$
\varphi=\{1, \log (x+2), \log (x+3), \ldots, \log (x+m)\}
$$

The aim is to get $\hat{Y}$ which is an estimate of $Y$ such that the square of the error is minimised. That is;

$$
\min \left\{\frac{1}{n} \sum_{i=1}^{n}\left(Y_{i}-\widehat{Y}_{i}\right)^{2}\right\} .
$$

Hence, the aim is to produce an estimate $\hat{Y}$ that gives minimal error $\varepsilon$ which can be represented as:

$$
\varepsilon=\min (Y-\widehat{Y}) \text {. }
$$

This final equation can be solved using least square optimization. Several useful analyses and applications of functional networks are found in the literature (de Jesus Rubio et al., 2012; Elsebakhi et al., 2015; Asafa et al., 2015).

\subsection{Proposed Hybrid K-means and Functional Networks}

The proposed hybrid implementation is shown in Fig. 3. The clusters serve as inputs to the FN. FN has been specifically chosen for the hybrid modelling as it has been shown to perform very well on both small and large data sets (Castillo et al., 2000). It is important to note that the clustering takes all the predictors into consideration to generate the clusters unlike the manual grouping based on only $\gamma_{A P I}$ grouping, which is done for some empirical correlations.

\section{Input: Get the data $X$ for clustering \\ 2. Data clustering with K-Means to get clusters, $D_{c}$, $c=1, \ldots, k$ where $k$ is total number of clusters \\ 3. BEGIN FN Network \\ 3. FOR $m=3$ to 10 \\ 4. Initialise the functional model e.g. $\varphi=$ $\left\{1, x, x^{2}, \ldots, x^{m}\right\}$ \\ 5. FOR each cluster $D_{c}$ compute the functional components $C$ \\ 6. END FOR $m$ loop \\ 7. END each cluster loop \\ 8. Combine and evaluate the prediction from the clusters.}

Fig. 3. Steps for the hybrid k-means and Functional Networks

All the four functional forms stated above have been tested with degrees between 3 and 10 which is sufficient in most cases (Castillo et al., 2000). The best chosen model is the one that gives both the minimum root mean squared error (RMSE) and minimum average absolute percentage relative error $\left(E_{a}\right)$.

\subsection{Experimental Work}

A total of approximately 1400 data points were available for the simulation. 327 data points were collected from different published papers (Al-Marhoun, 1988; Omar \& Todd, 1993; Dokla \& Osman, 1992; Bello et al., 2008). The remaining data are unpublished and they are from different sources such as GeoMark Research and Shell Company. The data comes from diverse crude oils across the globe.

In the hybrid systems, four input clusters have been generated which are passed to the FN. All the stated learning functions were tested and the polynomial function of degrees three to five gave the best results for both the hybrid KMeans+FN and the standalone FN. For the ANN model, different activation functions, number of hidden layers and neurons were explored. The best ANN model has been achieved with sigmoid activation, one hidden layer and ten neurons. The input variables for both $P_{b}$ and $B_{o b}$ are $R_{s}, \gamma_{g}, \gamma_{A P I}$ and $T$.

\section{Results and Discussion}

Simulation results of hybrid K-Means+FN for both $P_{b}$ and $B_{o b}$ are presented and compared with the standalone FN, ANN, popular and recently proposed empirical correlations. Mainly, two statistical error measures, $R M S E$ and $E_{a}$ are used to compare the prediction capability of the models. Whenever there 
is a tie between these two, then $E_{\text {max }}$ and $C C$ are used respectively for comparison. The lower the error parameters: $R M S E, E_{a}$ and $E_{\text {max }}$, the better is the model. On the other hand, the higher the $C C$, the better is the performance of the model.

\subsection{Comparison of Hybrid K-Means $+F N$ with other Models}

For $P_{b}$ prediction, the proposed hybrid K-Means+FN model gives both minimum RMSE (344.8264) and $E_{a}$ (11.0829) as shown in Table 2. K-Means+FN hybrid model also has the least $E_{\max }(81.8879)$ which implies that it has the least tendency of over-fitting. Likewise, K-Means $+\mathrm{FN}$ has the highest $C C$ $(0.9652)$ which is an indication that its predicted output are more correlated with the target than others.
Among all these compared models for $P_{b}$ prediction, the least performance is given by the correlation of (Jarrahian et al., 2015). From the results, both ANN and standalone FN also perform better than all the listed empirical correlations.

The results for the $B_{o b}$ prediction are shown in Table 3 . Clearly, the hybrid K-Means $+\mathrm{FN}$ gives the best performance with the least RMSE (0.0489) and $E_{a}$ (1.3856). Also the KMeans+FN hybrid has the minimum $E_{\max }$ (31.8356) and maximum $C C$ (0.9807). A very significant improvement in performance can be seen in the hybrid system compared to both ANN and standalone FN. However, both ANN and standalone FN trail one of the correlations (Petrosky Jr \& Farshad, 1998) in performance with respect to both $R M S E$ and $E_{a}$.

Table 2

Performances of different prediction methods for bubblepoint pressure

\begin{tabular}{|l|c|c|c|c|c|c|c|}
\hline Prediction Method & $\mathbf{C C}$ & $\mathbf{R M S E}$ & $\boldsymbol{E}_{\boldsymbol{r}}$ & $\boldsymbol{E}_{\boldsymbol{a}}$ & $\boldsymbol{E}_{\boldsymbol{m i n}}$ & $\boldsymbol{E}_{\max }$ & SD \\
\hline Standing (1947) & 0.9057 & 616.2760 & -3.4038 & 17.5340 & 0.0190 & 548.9583 & 0.3414 \\
\hline Al-Marhoun (1988) & 0.9187 & 812.2788 & -12.9768 & 20.0810 & 0.0084 & 388.6760 & 0.2222 \\
\hline Vazquez \& Beggs (1980) & 0.9091 & 765.8636 & -13.0168 & 20.6291 & 0.0212 & 603.2238 & 0.4345 \\
\hline Kartoatmodjo \& Schmidt (1991) & 0.8911 & 882.2240 & -11.1193 & 21.9222 & 0.0095 & 602.7154 & 0.4991 \\
\hline Dokla \& Osman (1992) & 0.8596 & 678.0152 & -5.3107 & 21.8189 & 0.0093 & 511.0192 & 0.7033 \\
\hline Petrosky Jr \& Farshad (1998) & 0.9347 & 793.0799 & 10.7982 & 39.7123 & 0.0022 & 1357.4589 & 0.0706 \\
\hline Velarde et al. (1999) & 0.9328 & 529.5030 & -5.2997 & 14.9442 & 0.0385 & 443.4241 & 0.1801 \\
\hline Al-Shammasi (2001) & 0.8962 & 579.3566 & -2.2827 & 18.9615 & 0.0334 & 576.1059 & 0.3534 \\
\hline Dindoruk \& Christman (2004) & 0.9247 & 540.7703 & -6.8919 & 18.2234 & 0.0300 & 467.5842 & 0.4716 \\
\hline Khamehchi et al. (2009) & 0.8947 & 1036.8969 & -24.4490 & 29.5174 & 0.0142 & 654.9375 & 0.8214 \\
\hline Arabloo et al. (2014) & 0.9104 & 551.5070 & 3.4806 & 16.5968 & 0.0001 & 461.4342 & 0.4964 \\
\hline Jarrahian et al. (2015) & 0.8597 & 2004.4451 & 73.7602 & 73.8764 & 25.8628 & 96.8158 & 0.1077 \\
\hline ANN & 0.9457 & 410.1738 & -1.2703 & 13.0577 & 0.0268 & 117.0041 & 0.6855 \\
\hline Standalone FN & 0.9522 & 390.5335 & -5.6662 & 14.5618 & 0.0057 & 154.4435 & 0.2733 \\
\hline Cluster + FN (Present work) & 0.9652 & 344.8264 & -2.9911 & 11.0829 & 0.0056 & 81.8879 & 0.1188 \\
\hline
\end{tabular}

Table 3

Performances of different prediction methods for oil formation volume factor at bubblepoint pressure

\begin{tabular}{|l|l|l|l|l|l|l|l|}
\hline Prediction Method & $\mathbf{C C}$ & $\mathbf{R M S E}$ & $\boldsymbol{E}_{\boldsymbol{r}}$ & $\boldsymbol{E}_{\boldsymbol{a}}$ & $\boldsymbol{E}_{\boldsymbol{m i n}}$ & $\boldsymbol{E}_{\boldsymbol{m a x}}$ & SD \\
\hline Standing (1947) & 0.9574 & 0.0744 & -0.4097 & 2.2852 & 0.0007 & 68.1578 & 0.1468 \\
\hline Vazquez \& Beggs (1980) & 0.9374 & 0.0866 & 0.4498 & 3.5164 & 0.0047 & 63.0057 & 0.2384 \\
\hline Al-Marhoun (1988) & 0.9611 & 0.0716 & 0.5751 & 2.2557 & 0.0007 & 53.0750 & 0.0499 \\
\hline Kartoatmodjo \& Schmidt (1991) & 0.9624 & 0.0679 & 0.3174 & 2.1148 & 0.0025 & 56.5184 & 0.0770 \\
\hline Dokla \& Osman (1992) & 0.9594 & 0.0759 & 0.5801 & 3.1316 & 0.0012 & 65.1839 & 0.0101 \\
\hline Al-Marhoun (1992) & 0.9646 & 0.0655 & 0.2331 & 1.9317 & 0.0038 & 57.4667 & 0.0962 \\
\hline Omar \& Todd (1993) & 0.9434 & 0.0896 & -0.3410 & 3.1626 & 0.0119 & 77.3916 & 0.2419 \\
\hline Almehaideb (1997) & 0.9472 & 0.0834 & 0.2608 & 3.8067 & 0.0055 & 54.3641 & 0.0846 \\
\hline Petrosky Jr \& Farshad (1998) & 0.9642 & 0.0654 & -0.0645 & 1.4851 & 0.0001 & 53.0785 & 0.0902 \\
\hline Al-Shammasi (2001) & 0.9490 & 0.0851 & -2.6715 & 3.7926 & 0.0206 & 67.3687 & 0.1473 \\
\hline Dindoruk \& Christman (2004) & 0.9086 & 0.1128 & 0.4307 & 3.3049 & 0.0012 & 95.3749 & 0.0869 \\
\hline Ikiensikimama \& Ajienka (2012) & 0.9629 & 0.0670 & -0.1210 & 2.1352 & 0.0013 & 55.7279 & 0.0258 \\
\hline
\end{tabular}




\begin{tabular}{|l|l|l|l|l|l|l|l|}
\hline Arabloo et al. (2014) & 0.9636 & 0.0671 & 0.4658 & 2.0435 & 0.0002 & 56.2252 & 0.1346 \\
\hline ANN & 0.9507 & 0.0830 & 1.4421 & 2.9658 & 0.0220 & 35.6288 & 0.1405 \\
\hline Standalone FN & 0.9699 & 0.0742 & -0.2664 & 2.0167 & 0.0095 & 50.4549 & 0.2843 \\
\hline Cluster + FN (Present work) & 0.9807 & 0.0489 & -0.0714 & 1.3856 & 0.0069 & 31.8356 & 0.0146 \\
\hline
\end{tabular}

\subsection{Sensitivity of Functional Network Parameters}

In this section, the sensitivity of the FN parameters in the overall performance of the hybrid K-Means+FN models is examined. In the previous section, the best achievable model for the hybrid has been used. Boxplots for the four most important statistical parameters are presented in Figs. 4-7.

It is noted that the performance of the hybrid models, largely influenced by the linear independent function and its degree for the $\mathrm{FN}$, can vary over a wide range. For both $P_{b}$ and $B_{o b}$, polynomial function of degrees three to five gave the best performances.

From the box plots in Figs. 4-7, it can be seen that performances of the hybrid K-Means $+\mathrm{FN}$ varied significantly for $C C, R M S E, E_{a}$ and $E_{\max }$. For $P_{b}, C C$ ranges from 0.9079 to $0.9652, R M S E$ from 344.8264 to $552.5147, E_{a}$ from $11.0829 \%$ to $31.2093 \%$ and $E_{\max }$ from $81.8879 \%$ to $2911.8493 \%$. For the $B_{o b}, C C$ ranges from 0.9614 to 0.9807 , $R M S E$ from 0.0489 to $0.0689, E_{a}$ from $1.3856 \%$ to $3.2082 \%$ and $E_{\max }$ from $31.8356 \%$ to $61.7369 \%$.
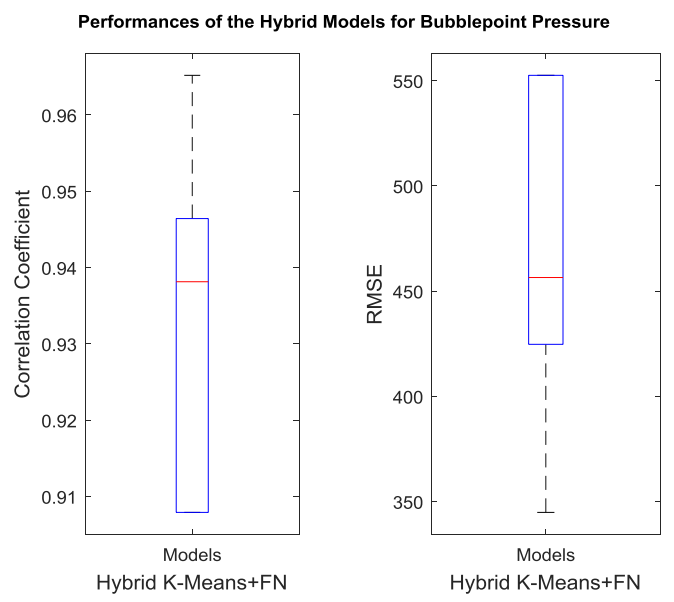

Fig. 4. $\quad P_{b}$ Boxplot for K-Means+FN and $C C /$ RMSE
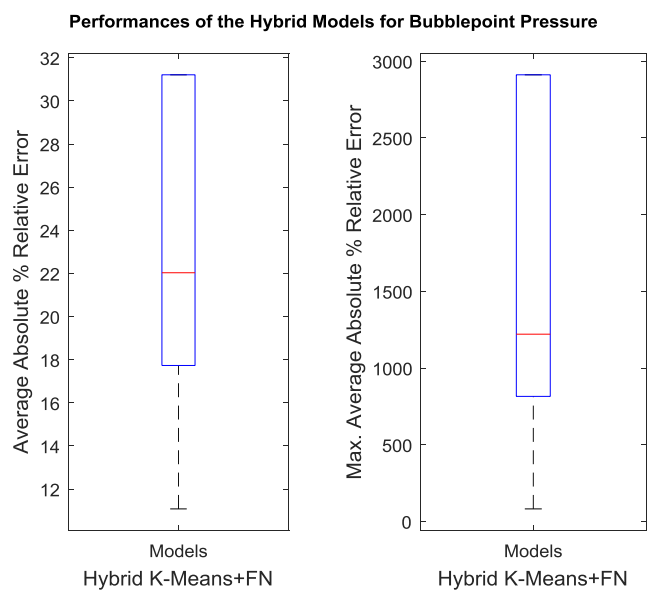

Fig. 5. $\quad P_{b}$ Boxplot for K-Means $+\mathrm{FN}$ and $E_{a} / E_{\max }$
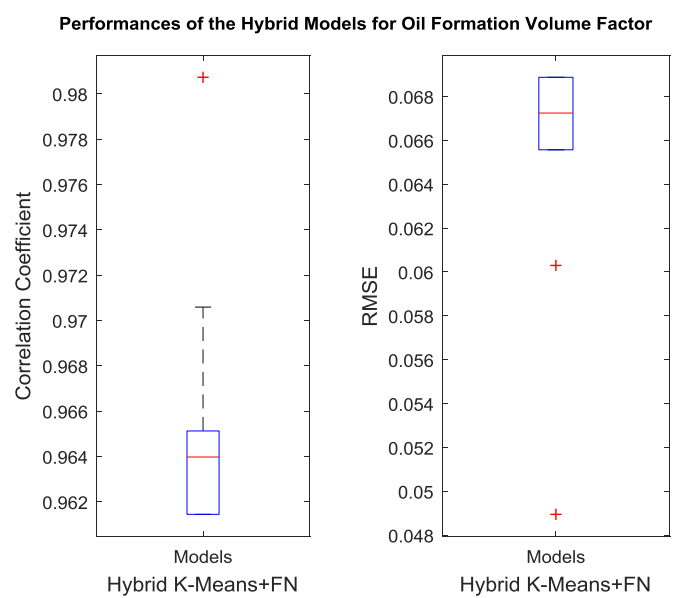

Fig. 6. $\quad B_{o b}$ Boxplot for K-Means+FN and RMSE/ $E_{a}$
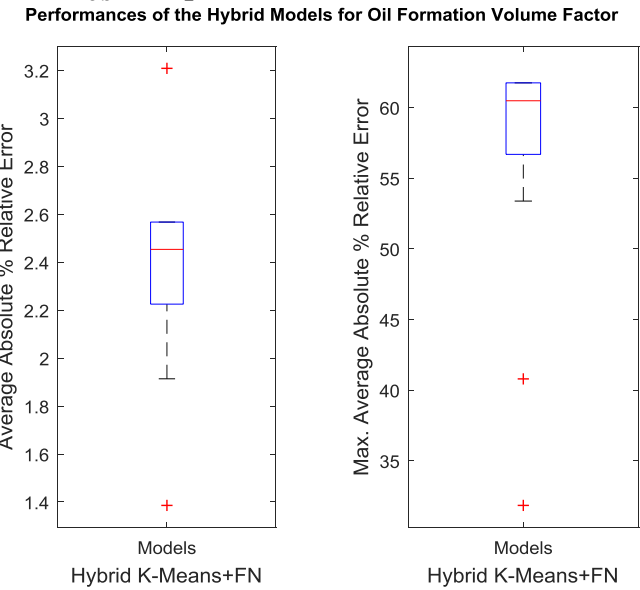

Fig. 7. $\quad B_{o b}$ Boxplot for K-Means+FN and $E_{a} / E_{\max }$ 


\section{Conclusion}

A hybrid system using K-Means clustering and functional networks has been developed to predict crude oil PVT based on worldwide data from a wide range of different crude oils with diverse thermodynamic properties. The clustering part of the hybrid is inspired by the common API grouping of crude oils.

Functional networks which resemble neural networks in architecture have been used for the actual prediction. The neurons in a functional network are functions defined by the modeller and it does not suffer from the 'black box' indictment, though it is more computationally demanding.

For the two PVT properties, $P_{b}$ and $B_{o b}$, that have been modelled in this work, the proposed hybrid system outperforms all the compared empirical correlations, feed forward neural network and standalone functional networks. The results show that the clustering of the data before prediction by the functional networks has significantly improve the results compared to the standalone functional networks. The attempt to solve the impact of imbalanced data set by pre-processing the data into clusters to prevent domination by most predominant crude oil with similar properties has shown its significance.

It is also clear that the learning parameters of the functional networks must be carefully selected to get a good high performance. It should be noted that different data sets in different problem will probably be modelled by different linear independent functions as expected. Hence, different ones must be explored in each problem to attain the model that appropriately captures the uncertainties in the input dataset.

Other PVT properties such as viscosity and gas-oil ratio can also benefit from this approach of clustering before prediction. Also, the K-Means clustering can be used with other ML techniques such as regression tree, SVM and extreme learning machine. Experimentation with other clustering techniques can also be considered in future research.

Lastly, the statistical descriptions of the data used in each cluster are given in the appendix along with the corresponding coefficients of the functional networks. New clusters and functional networks can be generated for significant data variability for new datasets.

\section{Appendix}

Statistical Measures for the performance Analysis

A.1. Average percent relative error

$$
E_{r}=\frac{1}{n} \sum_{1}^{n} E_{i}
$$

Where,

$$
\begin{aligned}
E_{i}=\left(\frac{X_{\text {exp }}-X_{\text {pred }}}{X_{\text {exp }}}\right)_{i} \times 100 \\
\quad i=1,2, \ldots, n
\end{aligned}
$$

A.2. Average absolute percent relative error

$$
E_{a}=\frac{1}{n} \sum_{1}^{n}\left|E_{i}\right|
$$

A.3. Maximum absolute percent relative error

$E_{\text {max }}=\max _{i}\left|E_{i}\right|$

\section{A.4. Standard Deviation}

$$
S D=\sqrt{\frac{1}{(n-1)} \sum_{i=1}^{n}\left(E_{i}-E_{r}\right)^{2}}
$$

Where,

A.5. Root mean squared

$$
E_{r}=\frac{1}{n} \sum_{i=1}^{n} E_{i} .
$$

$$
R M S E=\left[\frac{1}{n} \sum_{i=1}^{n} E_{i}\right]^{0.5}
$$

B. Statistical Description of Data in the Clusters

Tables B1-B4 show the mean (Mean), minimum (Min), Maximum (Max) and standard deviation (SD) of the data in the four clusters.

Table B.1

First Cluster (181 data points)

\begin{tabular}{|c|l|l|l|l|}
\hline Variable & Mean & Min & Max & SD \\
\hline$R_{s}$ & 1389.232 & 628.000 & 2637.000 & 452.535 \\
\hline$\gamma_{g}$ & 0.707 & 0.592 & 0.899 & 0.085 \\
\hline$Y_{A P I}$ & 37.926 & 22.300 & 48.200 & 4.283 \\
\hline $\mathrm{T}$ & 208.838 & 114.000 & 300.000 & 32.599 \\
\hline$P_{b}$ & 4649.996 & 3796.00 & 7142.700 & 669.333 \\
\hline$B_{o b}$ & 1.737 & 1.269 & 2.588 & 0.296 \\
\hline
\end{tabular}

Table B.2

Second Cluster (375 data points)

\begin{tabular}{|c|l|l|l|l|}
\hline Variable & Mean & Min & Max & SD \\
\hline$R_{s}$ & 256.273 & 10.000 & 840.000 & 150.634 \\
\hline$\gamma_{g}$ & 0.951 & 0.556 & 1.367 & 0.179 \\
\hline$Y_{A P I}$ & 32.341 & 11.600 & 55.000 & 6.926 \\
\hline $\mathrm{T}$ & 163.000 & 74.000 & 275.000 & 48.248 \\
\hline$P_{b}$ & 940.504 & 70.000 & 1593.000 & 430.858 \\
\hline$B_{o b}$ & 1.183 & 1.030 & 1.593 & 0.102 \\
\hline
\end{tabular}

Table B.3

Third Cluster (379 data points)

\begin{tabular}{|c|l|l|l|l|}
\hline Variable & Mean & Min & Max & SD \\
\hline$R_{s}$ & 872.562 & 60.0 & 1870.000 & 334.794 \\
\hline$\gamma_{g}$ & 0.744 & 0.570 & 1.101 & 0.104 \\
\hline$Y_{A P I}$ & 36.457 & 17.40 & 50.400 & 6.894 \\
\hline $\mathrm{T}$ & 189.219 & 100.0 & 327.000 & 43.938 \\
\hline$P_{b}$ & 3240.123 & 2559.0 & 3987.0 & 345.399 \\
\hline$B_{o b}$ & 1.487 & 1.075 & 2.088 & 0.222 \\
\hline
\end{tabular}

Table B.4

Fourth Cluster (465 data points)

\begin{tabular}{|c|l|l|l|l|}
\hline Variable & Mean & Min & Max & SD \\
\hline$R_{s}$ & 627.180 & 50.000 & 1602.000 & 237.920 \\
\hline$\gamma_{g}$ & 0.821 & 0.578 & 1.298 & 0.127 \\
\hline
\end{tabular}




\begin{tabular}{|l|l|l|l|l|}
\hline$Y_{A P I}$ & 37.825 & 15.900 & 53.400 & 7.014 \\
\hline $\mathrm{T}$ & 173.831 & 80.000 & 302.000 & 48.703 \\
\hline$P_{b}$ & 2158.456 & 1441.0 & 2800.00 & 336.627 \\
\hline$B_{o b}$ & 1.378 & 1.083 & 1.884 & 0.152 \\
\hline
\end{tabular}

$\mathrm{D} 4=[1.7900415134 .76633 E-04-0.044052727-$ $0.868929191-5.58737 E-040.061333617-$ $7.7291 E-077.55122 E-050.119961335-$ $0.0200828511 .19762 E-104.82085 E-$ $074.47238 E-04-4.10756 E-05-4.10455 E-$ $071.47728 E-08]$

C. FN Coefficients for $P_{b}$ prediction

The coefficients for the four clusters are given by $\mathrm{C} 1-\mathrm{C} 4$ respectively.

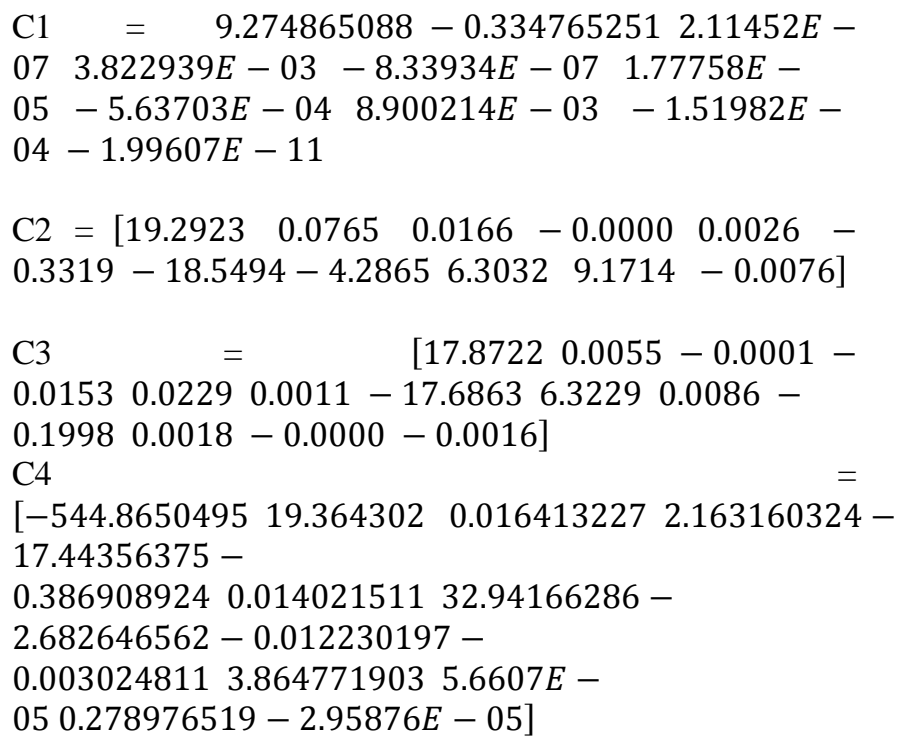

D. FN Coefficients for $B_{o b}$ prediction

The coefficients for the four clusters are given by D1-D4 respectively.

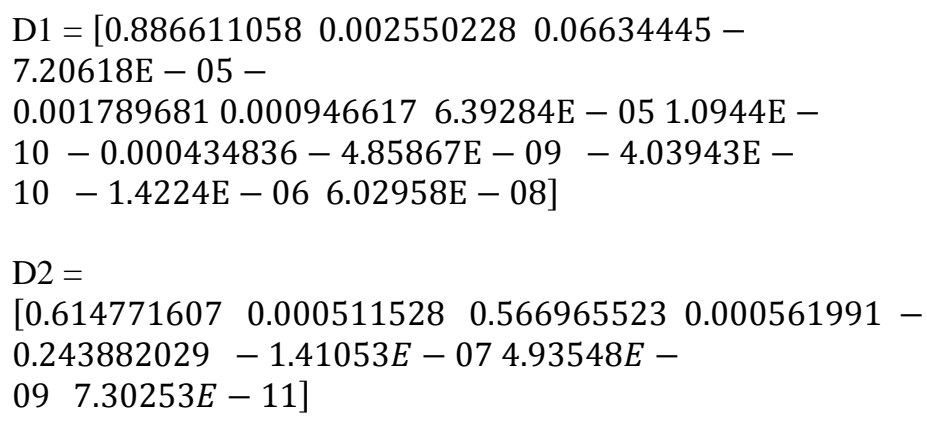

\section{Acknowledgment}

Oloso Munirudeen thanks the Petroleum Technology Development Fund, Nigeria, for sponsoring his $\mathrm{PhD}$ research at the University of Portsmouth. Special thanks also go to the Shell Petroleum Development Company, Nigeria, GeoMark Research and Professor T.A Blasingame of Texas A\& M University for supplying part of the data used in this work.

\section{References}

Al-Marhoun, M. A. (1988). PVT correlations for Middle East crude oils. Journal of Petroleum Technology, 40(5), 650-666.

Al-Marhoun, M. A. (1992). New Correlation for formation Volume Factor of oil and gas Mixtures. Journal of Canadian Petroleum Technology, 31(3), 22-26.

Al-Shammasi, A. A. (2001). A review of bubblepoint pressure and oil formation volume factor correlations. SPE Reservoir Evaluation \& Engineering, 4(2), 146160.

Aljarah, I., Faris, H., Mirjalili, S., \& Al-Madi, N. (2016). Training radial basis function networks using biogeography-based optimizer. Neural Computing and Applications, 1-25.

Almehaideb, R. A. (1997). Improved PVT correlations for UAE crude oils. In Middle east oil show and conference. Society of Petroleum Engineers.

Arabloo, M., Amooie, M.-A., Hemmati-Sarapardeh, A., Ghazanfari, M.-H., \& Mohammadi, A. H. (2014). Application of constrained multi-variable search methods for prediction of PVT properties of crude oil systems. Fluid Phase Equilibria, 363, 121-130.

Asafa, T. B., Adeniran, A. A., \& Olatunji, S. O. (2015). Functional networks models for rapid characterization of thin films: An application to ultrathin polycrystalline silicon germanium films. Applied Soft Computing, 28, $11-18$.

Bello, O. O., Reinicke, K. M., \& Patil, P. A. (2008). Comparison of the performance of empirical models used for the prediction of the PVT properties of crude oils of the niger delta. Petroleum Science and Technology, 26(5), 593-609.

Castillo, E. (1998). Functional networks. Neural Processing Letters, 7(3), 151-159.

Castillo, E., Cobo, A., Gutiérrez, J. M., \& Pruneda, E. (2000). 
Functional Networks: A New Network- Based Methodology. Computer-Aided Civil and Infrastructure Engineering, 15(2), 90-106.

Chairez, I. (2016). Adaptive Neural Network Nonparametric Identifier With Normalized Learning Laws. IEEE Transactions on Neural Networks and Learning Systems.

Dandekar, A. Y. (2013). Petroleum reservoir rock and fluid properties. CRC press.

De Ghetto, G., Paone, F., \& Villa, M. (1995). Pressurevolume-temperature correlations for heavy and extra heavy oils. In SPE International Heavy Oil Symposium. Society of Petroleum Engineers.

de Jesús Rubio, J. (2016). Least square neural network model of the crude oil blending process. Neural Networks, 78, 88-96.

de Jesús Rubio, J., Elias, I., Cruz, D. R., \& Pacheco, J. (2017). Uniform stable radial basis function neural network for the prediction in two mechatronic processes. Neurocomputing, 227, 122-130.

de Jesus Rubio, J., Salazar, M., Gomez, A. D., \& Lugo, R. (2012). Modeling of the relative humidity via functional networks and control of the temperature via classic controls for a bird incubator. Neural Computing and Applications, 21(7), 1491-1500.

Dietterich, T. G. (2000). Ensemble methods in machine learning. In Multiple classifier systems (pp. 1-15). Springer.

Dindoruk, B., \& Christman, P. G. (2004). PVT Properties and Viscosity Correlations for Gulf of Mexico Oils. SPE Reservoir Evaluation \& Engineering, 7(6), 427-437.

Dokla, M., \& Osman, M. (1992). Correlation of PVT Properties for UAE Crudes (includes associated papers 26135 and 26316). SPE Formation Evaluation, 7(1), 41-46.

El-Sebakhy, E. A., Sheltami, T., Al-Bokhitan, S. Y., Shaaban, Y., Raharja, P. D., \& Khaeruzzaman, Y. (2007). Support vector machines framework for predicting the PVT properties of crude oil systems. In SPE Middle East Oil and Gas Show and Conference. Society of Petroleum Engineers.

Elsebakhi, E., Lee, F., Schendel, E., Haque, A., Kathireason, N., Pathare, T., ... Al-Ali, R. (2015). Large-scale machine learning based on functional networks for biomedical big data with high performance computing platforms. Journal of Computational Science, 11, 6981.

Gharbi, R. B., Elsharkawy, A. M., \& Karkoub, M. (1999). Universal neural-network-based model for estimating the PVT properties of crude oil systems. Energy \& Fuels, 13(2), 454-458.

Hajizadeh, Y. (2007). Intelligent prediction of reservoir fluid viscosity. In Production and Operations Symposium. Society of Petroleum Engineers.

Ikiensikimama, S. S., \& Ajienka, J. A. (2012). Impact of PVT correlations development on hydrocarbon accounting: the case of the Niger Delta. Journal of Petroleum
Science and Engineering, 81, 80-85.

Jain, A. K. (2010). Data clustering: 50 years beyond Kmeans. Pattern Recognition Letters, 31(8), 651-666.

Jarrahian, A., Moghadasi, J., \& Heidaryan, E. (2015). Empirical estimating of black oils bubblepoint (saturation) pressure. Journal of Petroleum Science and Engineering, 126, 69-77.

Kartoatmodjo, T. R. S., \& Schmidt, Z. (1991). New correlations for crude oil physical properties.

Kartoatmodjo, T., \& Schmidt, Z. (1994). Large data bank improves crude physical property correlations. Oil and Gas Journal;(United States), 92(27).

Khamehchi, E., Rashidi, F., Rasouli, H., \& Ebrahimian, A. (2009). Novel empirical correlations for estimation of bubble point pressure, saturated viscosity and gas solubility of crude oils. Petroleum Science, 6(1), 8690.

Khoukhi, A., Oloso, M., Elshafei, M., Abdulraheem, A., \& Al-Majed, A. (2011). Support vector regression and functional networks for viscosity and gas/oil ratio curves estimation. International Journal of Computational Intelligence and Applications, 10(3), 269-293. http://doi.org/10.1142/S1469026811003100

Liu, Q., Yin, J., Leung, V. C. M., Zhai, J.-H., Cai, Z., \& Lin, J. (2016). Applying a new localized generalization error model to design neural networks trained with extreme learning machine. Neural Computing and Applications, 27(1), 59-66.

Omar, M. I., \& Todd, A. C. (1993). Development of new modified black oil correlations for Malaysian crudes. In SPE Asia Pacific oil and gas conference. Society of Petroleum Engineers.

Osman, E.-S. A., \& Al-Marhoun, M. A. (2005). Artificial neural networks models for predicting PVT properties of oil field brines. In SPE Middle East Oil and Gas Show and Conference. Society of Petroleum Engineers.

Petrosky Jr, G. E., \& Farshad, F. (1998). Pressure-volumetemperature correlations for Gulf of Mexico crude oils. SPE Reservoir Evaluation \& Engineering, 1(5), 416420.

Ramyachitra, D., \& Manikandan, P. (2014). Imbalanced Dataset Classification and Solutions: A Review. International Journal of Computing and Business Research (IJCBR), 5, 2229-6166.

Standing, M. B. (1947). A pressure-volume-temperature correlation for mixtures of California oils and gases. In Drilling and Production Practice. American Petroleum Institute.

Standing, M. B. (1977). Volumetric and phase behavior of oil field hydrocarbon systems: Society of Petroleum Engineers of AIME. Dallas, Texas.

Talebi, R., Ghiasi, M. M., Talebi, H., Mohammadyian, M., Zendehboudi, S., Arabloo, M., \& Bahadori, A. (2014). Application of soft computing approaches for modeling saturation pressure of reservoir oils. Journal of Natural Gas Science and Engineering, 20, 8-15.

Vazquez, M., \& Beggs, H. D. (1980). Correlations for fluid 
physical property prediction. Journal of Petroleum Technology, 32(6), 968-970.

Velarde, J., Blosingame, T. A., \& McCain Jr, W. D. (1999). Correlation of Black Oil Properties at Pressures Below Bubble Point Pressure--A New Approach (97-93). Journal of Canadian Petroleum Technology, 38(13), 62. 